\title{
Positive Solutions for Third-Order $p$-Laplacian Functional Dynamic Equations on Time Scales
}

\author{
Wen Guan and Da-Bin Wang \\ Department of Applied Mathematics, Lanzhou University of Technology, Lanzhou, Gansu 730050, China \\ Correspondence should be addressed to Da-Bin Wang; wangdb@lut.cn
}

Received 6 June 2014; Accepted 11 July 2014; Published 4 August 2014

Academic Editor: Hui-Sheng Ding

Copyright ( 2014 W. Guan and D.-B. Wang. This is an open access article distributed under the Creative Commons Attribution License, which permits unrestricted use, distribution, and reproduction in any medium, provided the original work is properly cited.

We study the following third-order $p$-Laplacian functional dynamic equation on time scales: $\left[\Phi_{p}\left(u^{\Delta \nabla}(t)\right)\right]^{\nabla}+a(t) f(u(t), u(\mu(t)))=$ $0, t \in(0, T)_{\mathrm{T}}, u(t)=\varphi(t), t \in[-r, 0]_{\mathrm{T}}, u^{\Delta}(0)=u^{\Delta \nabla}(T)=0$, and $u(T)+B_{0}\left(u^{\Delta}(\eta)\right)=0$. By applying the Five-Functional Fixed Point Theorem, the existence criteria of three positive solutions are established.

\section{Introduction}

Recently, much attention has been paid to the existence of positive solutions for the boundary value problems with $p$ Laplacian operator on time scales; for example, see [1-22] and the references therein. But, to the best of our knowledge, there is not much concerning $p$-Laplacian functional dynamic equations on time scales $[6,12-14,19,21,22]$, especially for the third-order $p$-Laplacian functional dynamic equations on time scales [14, 22].

In [14], Song and Gao were concerned with the existence of positive solutions for the $p$-Laplacian functional dynamic equation on time scales:

$$
\begin{gathered}
{\left[\Phi_{p}\left(u^{\Delta \nabla}(t)\right)\right]^{\nabla}+a(t) f(u(t), u(\mu(t)))=0, \quad t \in(0, T)_{\mathrm{T}},} \\
u(t)=\varphi(t), \quad t \in[-r, 0]_{\mathrm{T}}, \\
u^{\Delta}(0)=u^{\Delta \nabla}(T)=0, \quad u(T)+B_{0}\left(u^{\Delta}(\eta)\right)=0,
\end{gathered}
$$

where $\eta \in(0, \rho(T))_{\mathrm{T}}$ and $\Phi_{p}(s)$ is $p$-Laplacian operator; that is, $\Phi_{p}(s)=|s|^{p-2} s, p>1,\left(\Phi_{p}\right)^{-1}=\Phi_{q}, 1 / p+1 / q=1$, and

$\left(\mathrm{C}_{1}\right) f:\left(\mathbb{R}^{+}\right)^{2} \rightarrow \mathbb{R}^{+}$is continuous;

$\left(\mathrm{C}_{2}\right) a: \mathrm{T} \rightarrow \mathbb{R}^{+}$is left dense continuous (i.e., $a \in$ $\left.C_{\mathbf{l d}}\left(\mathbf{T}, \mathbb{R}^{+}\right)\right)$and does not vanish identically on any closed subinterval of $[0, T]$, where $C_{\mathbf{l d}}\left(\mathbf{T}, \mathbb{R}^{+}\right)$denotes the set of all left dense continuous functions from $\mathbf{T}$ to $\mathbb{R}^{+}$;

$\left(\mathrm{C}_{3}\right) \varphi:[-r, 0]_{\mathrm{T}} \rightarrow \mathbb{R}^{+}$is continuous and $r>0$;

$\left(\mathrm{C}_{4}\right) \mu:[0, T]_{\mathrm{T}} \rightarrow[-r, T]_{\mathrm{T}}$ is continuous, $\mu(t) \leq 0$ for all $t$

$\left(C_{5}\right) B_{0}: \mathbb{R} \rightarrow \mathbb{R}$ is continuous and satisfies the condition that there are $A \geq B \geq 0$ such that

$$
B v \leq B_{0}(v) \leq A v, \quad \forall \mathbb{R} .
$$

The existence of two positive solutions to problem (1) was obtained by using a double fixed point theorem due to Avery et al. [23] in a cone.

In [22], Wang and Guan considered the existence of positive solutions to problem (1) by applying the well-known Leggett-Williams Fixed Point Theorem.

Motivated by $[14,22]$, we will show that problem (1) has at least three positive solutions by means of the Five-Functional Fixed Point Theorem [24] (which is a generalization of the Leggett-Williams Fixed Point Theorem [25]). It is worth noting that the Five-Functional Fixed Point Theorem is used extensively in yielding three solutions for BVPs of differential equations, difference equations, and/or dynamic equations on time scales; see $[6,26,27]$ and references therein.

Throughout this work we assume knowledge of time scales and time-scale notation, first introduced by Hilger [28]. 
For more on time scales, please see the texts by Bohner and Peterson $[29,30]$.

In the remainder of this section, we state the following theorem, which is crucial to our proof.

Let $\gamma, \beta, \theta$ be nonnegative, continuous, and convex functionals on $P$ and let $\alpha, \psi$ be nonnegative, continuous, and concave functionals on $P$. Then, for nonnegative real numbers $h, a, b, d$, and $c$, we define the convex sets

$$
\begin{gathered}
P(\gamma, c)=\{x \in P: \gamma(x)<c\}, \\
P(\gamma, \alpha, a, c)=\{x \in P: a \leq \alpha(x), \gamma(x) \leq c\}, \\
Q(\gamma, \beta, d, c)=\{x \in P: \beta(x) \leq d, \gamma(x) \leq c\}, \\
P(\gamma, \theta, \alpha, a, b, c)=\{x \in P: a \leq \alpha(x), \theta(x) \leq b, \gamma(x) \leq c\}, \\
Q(\gamma, \beta, \psi, h, d, c) \\
=\{x \in P: h \leq \psi(x), \beta(x) \leq d, \gamma(x) \leq c\} .
\end{gathered}
$$

Theorem 1 (see [24]). Let $P$ be a cone in a real Banach space $E$. Suppose there exist positive numbers $c$ and $M$; nonnegative, continuous, and concave functionals $\alpha$ and $\psi$ on $P$; and nonnegative, continuous, and convex functionals $\gamma, \beta$, and $\theta$ on $P$, with

$$
\alpha(x) \leq \beta(x), \quad\|x\| \leq M \gamma(x)
$$

for all $x \in \overline{P(\gamma, c)}$. Suppose

$$
F: \overline{P(\gamma, c)} \longrightarrow \overline{P(\gamma, c)}
$$

is completely continuous and there exist nonnegative numbers $h, a, k, b$, with $0<a<b$ such that

(i) $\{x \in P(\gamma, \theta, \alpha, b, k, c): \alpha(x)>b\} \neq \varnothing$ and $\alpha(F x)>b$ for $x \in P(\gamma, \theta, \alpha, b, k, c)$;

(ii) $\{x \in Q(\gamma, \beta, \psi, h, a, c): \beta(x)<a\} \neq \varnothing$ and $\beta(F x)<a$ for $x \in Q(\gamma, \beta, \psi, h, a, c)$;

(iii) $\alpha(F x)>b$ for $x \in P(\gamma, \alpha, b, c)$ with $\theta(F x)>k$;

(iv) $\beta(F x)<a$ for $x \in Q(\gamma, \beta, a, c)$ with $\psi(F x)<h$.

Then $F$ has at least three fixed points $x_{1}, x_{2}, x_{3} \in \overline{P(\gamma, c)}$ such that

$$
\begin{gathered}
\beta\left(x_{1}\right)<a, \quad b<\alpha\left(x_{2}\right), \\
a<\beta\left(x_{3}\right) \quad \text { with } \alpha\left(x_{3}\right)<b .
\end{gathered}
$$

\section{Existence of Three Positive Solutions}

We note that $u(t)$ is a solution of BVP (1) if and only if

$u(t)$

$$
=\left\{\begin{array}{l}
\int_{0}^{T}(T-s) \Phi_{q}\left(\int_{0}^{s} a(r) f(u(r), u(\mu(r))) \nabla r\right) \nabla s \\
-B_{0}\left(\int_{0}^{\eta} \Phi_{q}\left(\int_{0}^{s}-a(r) f(u(r), u(\mu(r))) \nabla r\right) \nabla s\right) \\
+\int_{0}^{t}(t-s) \Phi_{q}\left(\int_{0}^{s}-a(r) f(u(r), u(\mu(r))) \nabla r\right) \nabla s, \\
\varphi(t), \quad t \in[0, T]_{\mathrm{T}}, \\
t \in[-r, 0]_{\mathrm{T}} .
\end{array}\right.
$$

Let $E=C_{\mathbf{l d}}\left([0, T]_{\mathrm{T}}, \mathbb{R}\right)$ be endowed with $\|u\|=$ $\sup _{t \in[0, T]_{T}}|u(t)|$, so $E$ is a Banach space. Define cone $P \subset E$ by

$$
\begin{aligned}
P=\{u \in E: & u \text { is concave and } \\
& \left.\quad \text { nonnegative valued on }[0, T]_{\mathrm{T}}, u^{\Delta}(0)=0\right\} .
\end{aligned}
$$

For each $u \in E$, extend $u(t)$ to $[-r, T]_{\mathrm{T}}$ with $u(t)=\varphi(t)$ for $t \in[-r, 0]_{\mathrm{T}}$.

Define $F: P \rightarrow E$ by

$(F u)(t)$

$$
=\int_{0}^{T}(T-s) \Phi_{q}
$$

$$
\begin{array}{r}
\times\left(\int_{0}^{s} a(r) f(u(r), u(\mu(r))) \nabla r\right) \nabla s \\
-B_{0}\left(\int_{0}^{\eta} \Phi_{q}\left(\int_{0}^{s}-a(r) f(u(r), u(\mu(r))) \nabla r\right) \nabla s\right) \\
+\int_{0}^{t}(t-s) \Phi_{q}\left(\int_{0}^{s}-a(r) f(u(r), u(\mu(r))) \nabla r\right) \nabla s, \\
t \in[0, T]_{\mathrm{T}} .
\end{array}
$$

We seek a point, $u_{1}$, of $F$ in the cone $P$. Define

$$
u(t)= \begin{cases}u_{1}(t), & t \in[0, T]_{\mathrm{T}}, \\ \varphi(t), & t \in[-r, 0]_{\mathrm{T}} .\end{cases}
$$

Then $u(t)$ denotes a positive solution of BVP (1).

We have the following results.

Lemma 2. Let $u \in P$, and then

(1) $F: P \rightarrow P$ is completely continuous;

(2) $u(t) \geq((T-t) / T)\|u\|$ for $t \in[0, T]_{\mathrm{T}}$;

(3) $u(t)$ is decreasing $[0, T]_{\mathrm{T}}$;

(4) $(T-\varsigma) u(\tau) \leq(T-\tau) u(\varsigma)$ for $0<\tau<\varsigma<T$ and $\tau, \varsigma \in \mathbf{T}$. 
Proof. (1)-(3) are Lemma 3.1 of [14]. It is easy to conclude that (4) is satisfied by the concavity of $u$.

Let $l \in \mathrm{T}$ be fixed such that $0<l<\eta<T$, and set

$$
\begin{aligned}
& Y_{1}=\left\{t \in[0, T]_{\mathrm{T}}: \mu(t)<0\right\} ; \\
& Y_{2}=\left\{t \in[0, T]_{\mathrm{T}}: \mu(t) \geq 0\right\} ; \\
& Y_{3}=Y_{1} \cap[0, l]_{\mathrm{T}} .
\end{aligned}
$$

Throughout this paper, we assume $Y_{3} \neq \varnothing$ and $\int_{Y_{3}} a(r) \nabla r>0$.

We define the nonnegative, continuous, and concave functionals $\alpha, \psi$ and the nonnegative, continuous, and convex functionals $\beta, \theta, \gamma$ on the cone $P$, respectively, as

$$
\begin{gathered}
\gamma(u)=\theta(u)=\max _{t \in[\eta, T]_{\mathrm{T}}} u(t)=u(\eta), \\
\alpha(u)=\min _{t \in[0, l]_{\mathrm{T}}} u(t)=u(l), \\
\beta(u)=\max _{t \in[l, T]_{\mathrm{T}}} u(t)=u(l) \\
\psi(u)=\min _{t \in[0, \eta]_{\mathrm{T}}} u(t)=u(\eta) .
\end{gathered}
$$

We observe that $\alpha(u)=\beta(u)$ for each $u \in P$.

In addition, by Lemma 2 , we have $\gamma(u)=u(\eta) \geq((T-$ $\eta) / T)\|u\|$. Hence $\|u\| \leq(T /(T-\eta)) \gamma(u)$ for all $u \in P$.

For convenience, we define

$$
\begin{gathered}
\mu=T(T+\eta+B) \Phi_{q}\left(\int_{0}^{T} a(r) \nabla r\right), \\
\delta=A \int_{Y_{3}} \Phi_{q}\left(\int_{0}^{s} a(r) \nabla r\right) \nabla s, \\
\lambda=T(T+l+B) \Phi_{q}\left(\int_{0}^{T} a(r) \nabla r\right) .
\end{gathered}
$$

We now state growth conditions on $f$ so that BVP (1) has at least three positive solutions.

Theorem 3. Let $0<a<((T-l) / T) b<\left((T-\eta)(T-l) / T^{2}\right) c$, $\mu b<\delta c$, and suppose that $f$ satisfies the following conditions:

$\left(\mathrm{H}_{1}\right) f(u, \varphi(s))<\Phi_{p}(c / \mu)$, if $0 \leq u \leq(T /(T-\eta)) c$, uniformly in $s \in[-r, 0]_{\mathrm{T}}$, and $f\left(u_{1}, u_{2}\right)<\Phi_{p}(c / \mu)$, if $0 \leq u_{i} \leq(T /(T-\eta)) c, i=1,2$;

$\left(\mathrm{H}_{2}\right) f(u, \varphi(s))>\Phi_{p}(b / \delta)$, if $b \leq u \leq(T /(T-\eta))^{2} b$, uniformly in $s \in[-r, 0]_{\mathrm{T}}$;

$\left(\mathrm{H}_{3}\right) f(u, \varphi(s))<\Phi_{p}(a / \lambda)$, if $0 \leq u \leq(T /(T-l)) a$, uniformly in $s \in[-r, 0]_{\mathrm{T}}$, and $f\left(u_{1}, u_{2}\right)<\Phi_{p}(a / \lambda)$, if $0 \leq u_{i} \leq(T /(T-l)) a, i=1,2$.

Then BVP (1) has at least three positive solutions of the form

$$
u(t)= \begin{cases}u_{i}(t), & t \in[0, T]_{\mathrm{T}}, \quad i=1,2,3, \\ \varphi(t), & t \in[-r, 0]_{\mathrm{T}},\end{cases}
$$

where $\max _{t \in[l, T]_{\mathrm{T}}} u_{1}(t)<a, \min _{t \in[0, l]_{\mathrm{T}}} u_{2}(t)>b$, and $a<$ $\max _{t \in[l, T]_{\mathrm{T}}} u_{3}(t)$ with $\min _{t \in[0, l]_{\mathrm{T}}} u_{3}(t)<b$.

Proof. Let $u \in \overline{P(\gamma, c)}$, and then $\gamma(u)=\max _{t \in[\eta, T]_{\mathrm{T}}} u(t)=$ $u(\eta) \leq c$, and consequently, $0 \leq u(t) \leq c$ for $t \in[\eta, T]_{\mathrm{T}}$. Since $u(\eta) \geq((T-\eta) / T) u(0)$, so $\|u\|=u(0) \leq(T /(T-\eta)) u(\eta) \leq$ $(T /(T-\eta)) c$, and this implies

$$
0 \leq u(t) \leq \frac{T}{T-\eta} c, \quad \text { for } t \in[0, T]_{\mathrm{T}} .
$$

From $\left(\mathrm{H}_{1}\right)$, we have

$$
\begin{aligned}
\gamma(F u) & (F u)(\eta) \\
= & \int_{0}^{T}(T-s) \Phi_{q}\left(\int_{0}^{s} a(r) f(u(r), u(\mu(r))) \nabla r\right) \nabla s \\
& -B_{0}\left(\int_{0}^{\eta} \Phi_{q}\left(\int_{0}^{s}-a(r) f(u(r), u(\mu(r))) \nabla r\right) \nabla s\right) \\
& +\int_{0}^{\eta}(\eta-s) \Phi_{q}\left(\int_{0}^{s}-a(r) f(u(r), u(\mu(r))) \nabla r\right) \nabla s \\
\leq & T \Phi_{q}\left(\int_{0}^{s} a(r) f(u(r), u(\mu(r))) \nabla r\right) \nabla s \\
& +B \int_{0}^{T} \Phi_{q}\left(\int_{0}^{s} a(r) f(u(r), u(\mu(r))) \nabla r\right) \nabla s \\
& +\int_{0}^{\eta} T \Phi_{q}\left(\int_{0}^{T} a(r) f(u(r), u(\mu(r))) \nabla r\right) \nabla s \\
= & T(T+\eta+B) \Phi_{q}\left[\int_{Y_{1}} a(r) f(u(r), \varphi(\mu(r))) \nabla r\right. \\
& \\
& \\
& \\
& \\
& \\
& \\
&
\end{aligned}
$$

Therefore

$$
F u \in \overline{P(\gamma, c)}
$$

We now turn to property (i) of Theorem 1 . Choosing $u \equiv$ $(T /(T-\eta)) b, k=(T /(T-\eta)) b$, it follows that

$$
\begin{aligned}
& \alpha(u)=u(l)=\frac{T}{T-\eta} b>b, \\
& \theta(u)=u(\eta)=\frac{T}{T-\eta} b=k, \\
& \gamma(u)=u(\eta)=\frac{T}{T-\eta} b<c,
\end{aligned}
$$


which shows that $\{u \in P(\gamma, \theta, \alpha, b, k, c): \alpha(u)>b\} \neq \varnothing$, and, for $u \in P(\gamma, \theta, \alpha, b,(T /(T-\eta)) b, c)$, we have

$$
b \leq u(t) \leq\left(\frac{T}{T-\eta}\right)^{2} b, \quad \text { for } t \in[0, l]_{\mathrm{T}} .
$$

From $\left(\mathrm{H}_{2}\right)$, we have

$$
\begin{aligned}
\alpha(F u) & \\
= & (F u)(l) \\
= & \int_{0}^{T}(T-s) \Phi_{q}\left(\int_{0}^{s} a(r) f(u(r), u(\mu(r))) \nabla r\right) \nabla s \\
& -B_{0}\left(\int_{0}^{\eta} \Phi_{q}\left(\int_{0}^{s}-a(r) f(u(r), u(\mu(r))) \nabla r\right) \nabla s\right) \\
& +\int_{0}^{l}(t-s) \Phi_{q}\left(\int_{0}^{s}-a(r) f(u(r), u(\mu(r))) \nabla r\right) \nabla s \\
\geq & -B_{0}\left(\int_{0}^{\eta} \Phi_{q}\left(\int_{0}^{s}-a(r) f(u(r), u(\mu(r))) \nabla r\right) \nabla s\right) \\
\geq & A \int_{0}^{\eta} \Phi_{q}\left(\int_{0}^{s} a(r) f(u(r), u(\mu(r))) \nabla r\right) \nabla s \\
\geq & A \int_{0}^{l} \Phi_{q}\left(\int_{0}^{s} a(r) f(u(r), u(\mu(r))) \nabla r\right) \nabla s \\
\geq & A \int_{Y_{3}} \Phi_{q}\left(\int_{0}^{s} a(r) f(u(r), \varphi(\mu(r))) \nabla r\right) \nabla s \\
> & A \int_{Y_{3}} \Phi_{q}\left(\int_{0}^{s} a(r) \nabla r\right) \nabla s \frac{b}{\delta}=b .
\end{aligned}
$$

We conclude that (i) of Theorem 1 is satisfied.

We next address (ii) of Theorem 1 . If we take $u \equiv((T-$ $\eta) / T) a, h=((T-\eta) / T) a$, then

$$
\begin{aligned}
& \gamma(u)=u(\eta)=\frac{T-\eta}{T} a<c, \\
& \psi(u)=u(\eta)=\frac{T-\eta}{T} a=h, \\
& \beta(u)=u(l)=\frac{T-\eta}{T} a<a .
\end{aligned}
$$

From this we know that $\{u \in Q(\gamma, \beta, \psi, h, a, c): \beta(u)<$ $a\} \neq \varnothing$. If $u \in Q(\gamma, \beta, \psi,((T-\eta) / T) a, a, c)$, then

$$
0 \leq u(t) \leq \frac{T}{T-l} a, \quad \text { for } t \in[0, T]_{\mathrm{T}} .
$$

From $\left(\mathrm{H}_{3}\right)$, we have

$$
\begin{aligned}
\beta(F u) & (F u)(l) \\
= & \int_{0}^{T}(T-s) \Phi_{q}\left(\int_{0}^{s} a(r) f(u(r), u(\mu(r))) \nabla r\right) \nabla s \\
& -B_{0}\left(\int_{0}^{\eta} \Phi_{q}\left(\int_{0}^{s}-a(r) f(u(r), u(\mu(r))) \nabla r\right) \nabla s\right) \\
& +\int_{0}^{l}(t-s) \Phi_{q}\left(\int_{0}^{s}-a(r) f(u(r), u(\mu(r))) \nabla r\right) \nabla s \\
\leq & \int_{0}^{T} T \Phi_{q}\left(\int_{0}^{s} a(r) f(u(r), u(\mu(r))) \nabla r\right) \nabla s \\
& +B \int_{0}^{T} \Phi_{q}\left(\int_{0}^{s} a(r) f(u(r), u(\mu(r))) \nabla r\right) \nabla s \\
& +\int_{0}^{l} T \Phi_{q}\left(\int_{0}^{T} a(r) f(u(r), u(\mu(r))) \nabla r\right) \nabla s \\
= & T(T+l+B) \Phi_{q}\left[\int_{Y_{1}} a(r) f(u(r), \varphi(\mu(r))) \nabla r\right. \\
< & \left.+\int_{Y_{2}} a(r) f(u(r), u(\mu(r))) \nabla r\right] \\
&
\end{aligned}
$$

Now we show that (iii) of Theorem 1 is satisfied. If $u \in$ $P(\gamma, \alpha, b, c)$ and $\theta(F u)=F u(\eta)>(T /(T-\eta)) b$, then

$$
\begin{aligned}
\alpha(F u) & \geq(F u)(l)=\frac{T-l}{T} F u(l) \geq \frac{T-l}{T} F u(\eta) \\
& >\frac{T-l}{T-\eta} b>b .
\end{aligned}
$$

Finally, if $u \in Q(\gamma, \beta, a, c)$ and $\psi(F u)=F u(\eta)<((T-$ $\eta) / T) a$, then from (4) of Lemma 2 we have

$$
\beta(F u)=F u(l) \leq \frac{T}{T-l} F u(l) \leq \frac{T}{T-\eta} F u(\eta)<a,
$$

which shows that condition (iv) of Theorem 1 is fulfilled.

Thus, all the conditions of Theorem 1 are satisfied. Hence, $F$ has at least three fixed points $u_{1}, u_{2}, u_{3}$ satisfying

$$
\begin{gathered}
\beta\left(u_{1}\right)<a, \quad b<\alpha\left(u_{2}\right), \\
a<\beta\left(u_{3}\right) \quad \text { with } \alpha\left(u_{3}\right)<b .
\end{gathered}
$$

Let

$$
u(t)= \begin{cases}u_{i}(t), & t \in[0, T]_{\mathrm{T}}, \quad i=1,2,3, \\ \varphi(t), & t \in[-r, 0]_{\mathrm{T}},\end{cases}
$$

which are three positive solutions of BVP (1). 


\section{Conflict of Interests}

The authors declare that there is no conflict of interests regarding the publication of this paper.

\section{Acknowledgments}

The authors thank the referees and the editors for their helpful comments and suggestions. Research was supported by the Postdoctoral Fund in China (Grant no. 2013M531717), the Excellent Young Teacher Training Program of Lanzhou University of Technology (Grant no. Q200907), and the Natural Science Foundation of Gansu Province of China (Grant no. 1310RJYA080).

\section{References}

[1] D. Anderson, R. Avery, and J. Henderson, "Existence of solutions for a one dimensional $p$-Laplacian on time-scales," Journal of Difference Equations and Applications, vol. 10, no. 10, pp. 889896, 2004.

[2] D. R. Anderson, "Existence of solutions for a first-order $p$ Laplacian BVP on time scales," Nonlinear Analysis: Theory, Methods \& Applications, vol. 69, no. 12, pp. 4521-4525, 2008.

[3] L. Bian, X. He, and H. Sun, "Multiple positive solutions of $m$ point BVPs for third-order $p$-Laplacian dynamic equations on time scales," Advances in Difference Equations, vol. 2009, Article ID 262857, 12 pages, 2009.

[4] A. Cabada, "Existence results for $p$-Laplacian boundary value problems on time scales," Advances in Difference Equations, vol. 11, Article ID 21819, 2006.

[5] A. Cabada, "Discontinuous functional $p$-Laplacian boundary value problems on time scales," International Journal of Difference Equations, vol. 2, no. 1, pp. 51-60, 2007.

[6] W. Guan, "Three positive solutions for $p$-Laplacian functional dynamic equations on time scales," Electronic Journal of Qualitative Theory of Differential Equations, No. 28, 7 pages, 2008.

[7] W. Han and S. Kang, "Multiple positive solutions of nonlinear third-order BVP for a class of $p$-Laplacian dynamic equations on time scales," Mathematical and Computer Modelling, vol. 49, no. 3-4, pp. 527-535, 2009.

[8] Z. M. He, "Double positive solutions of three-point boundary value problems for $p$-Laplacian dynamic equations on time scales," Journal of Computational and Applied Mathematics, vol. 182, no. 2, pp. 304-315, 2005.

[9] Z. He and X. Jiang, "Triple positive solutions of boundary value problems for $p$-Laplacian dynamic equations on time scales," Journal of Mathematical Analysis and Applications, vol. 321, no. 2, pp. 911-920, 2006.

[10] Z. M. He and Z. Long, "Three positive solutions of three-point boundary value problems for $p$-Laplacian dynamic equations on time scales," Nonlinear Analysis: Theory, Methods \& Applications, vol. 69, no. 2, pp. 569-578, 2008.

[11] S. Hong, "Triple positive solutions of three-point boundary value problems for $p$-Laplacian dynamic equations on time scales," Journal of Computational and Applied Mathematics, vol. 206, no. 2, pp. 967-976, 2007.

[12] C. Song and C. Xiao, "Positive solutions for P-Laplacian functional dynamic equations on time scales," Nonlinear Analysis: Theory, Methods \& Applications, vol. 66, no. 9, pp. 1989-1998, 2007.
[13] C. X. Song and P. X. Weng, "Multiple positive solutions for $p$ Laplacian functional dynamic equations on time scales," Nonlinear Analysis: Theory, Methods \& Applications, vol. 68, no. 1, pp. 208-215, 2008.

[14] C. Song and X. Gao, "Positive solutions for third-order $p$-Laplacian functional dynamic equations on time scales," Boundary Value Problems, vol. 2011, Article ID 279752, 2011.

[15] Y. Su, W. Li, and H. Sun, "Positive solutions of singular $p$ Laplacian BVPs with sign changing nonlinearity on time scales," Mathematical and Computer Modelling, vol. 48, no. 5-6, pp. 845$858,2008$.

[16] H. Sun, L. Tang, and Y. Wang, "Eigenvalue problem for $p$-Laplacian three-point boundary value problems on time scales," Journal of Mathematical Analysis and Applications, vol. 331, no. 1, pp. 248-262, 2007.

[17] H. Sun and W. Li, "Existence theory for positive solutions to one-dimensional $p$-Laplacian boundary value problems on time scales," Journal of Differential Equations, vol. 240, no. 2, pp. 217-248, 2007.

[18] D. B. Wang, "Existence, multiplicity and infinite solvability of positive solutions for $p$-Laplacian dynamic equations on time scales," Electronic Journal of Differential Equations, no. 96, 10 pages, 2006.

[19] D. B. Wang, "Three positive solutions for $p$-Laplacian functional dynamic equations on time scales," Electronic Journal of Differential Equations, vol. 2007, no. 95, pp. 1-9, 2007.

[20] D. B. Wang, “Three positive solutions of three-point boundary value problems for $p$-Laplacian dynamic equations on time scales," Nonlinear Analysis: Theory, Methods \&Applications, vol. 68, no. 8, pp. 2172-2180, 2008.

[21] D. Wang and W. Guan, "Multiple positive solutions for $p$ Laplacian functional dynamic equations on time scales," Taiwanese Journal of Mathematics, vol. 12, no. 9, pp. 2327-2340, 2008.

[22] D. B. Wang and W. Guan, "Multiple positive solutions for third-order $p$-Laplacian functional dynamic equations on time scales," Advances in Difference Equations, vol. 2014, article 145, 2014.

[23] R. I. Avery, C. J. Chyan, and J. Henderson, "Twin solutions of boundary value problems for ordinary differential equations and finite difference equations," Computers \& Mathematics with Applications, vol. 42, no. 3-5, pp. 695-704, 2001.

[24] R. Avery and J. Henderson, "Existence of three positive pseudosymmetric solutions for a one-dimensional $p$-Laplacian," Journal of Mathematical Analysis and Applications, vol. 277, no. 2, pp. 395-404, 2003.

[25] R. W. Leggett and L. R. Williams, "Multiple positive fixed points of nonlinear operators on ordered Banach spaces," Indiana University Mathematics Journal, vol. 28, no. 4, pp. 673-688, 1979.

[26] J. Li and J. Shen, "Existence of three positive solutions for boundary value problems with $p$-Laplacian," Journal of Mathematical Analysis and Applications, vol. 311, no. 2, pp. 457-465, 2005.

[27] D. Wang and W. Guan, "Three positive solutions of boundary value problems for $P$-Laplacian difference equations," Computers \& Mathematics with Applications, vol. 55, no. 9, pp. 19431949, 2008.

[28] S. Hilger, "Analysis on measure chains: a unified approach to continuous and discrete calculus," Results in Mathematics, vol. 18, no. 1-2, pp. 18-56, 1990. 
[29] M. Bohner and A. Peterson, Dynamic Equations on Time Scales, An Introduction with Applications, Birkhäuser, Boston, Mass, USA, 2001.

[30] M. Bohner and A. Peterson, Advances in Dynamic Equations on Time Scales, Birkhäuser, Boston, Mass, USA, 2003. 


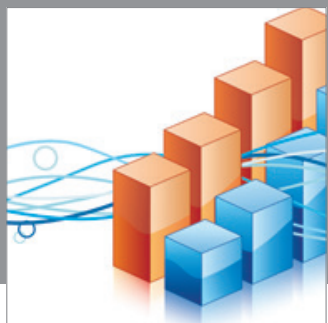

Advances in

Operations Research

mansans

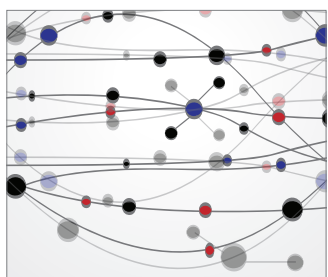

The Scientific World Journal
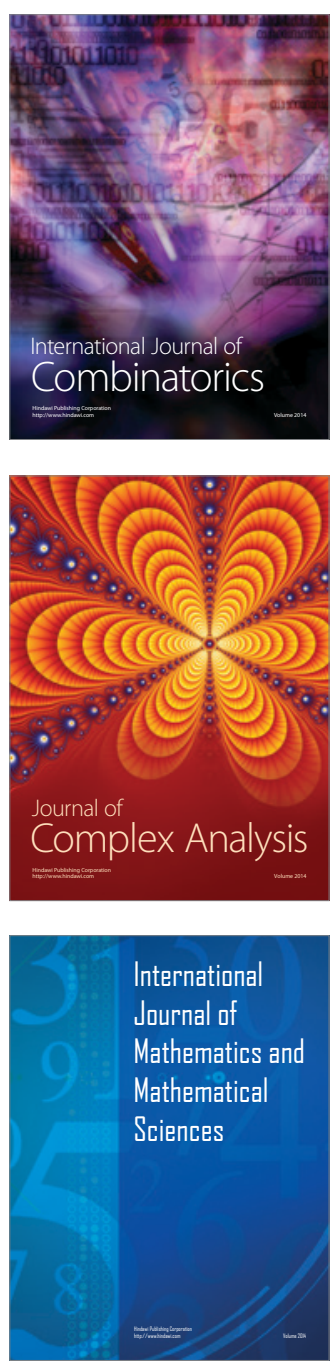
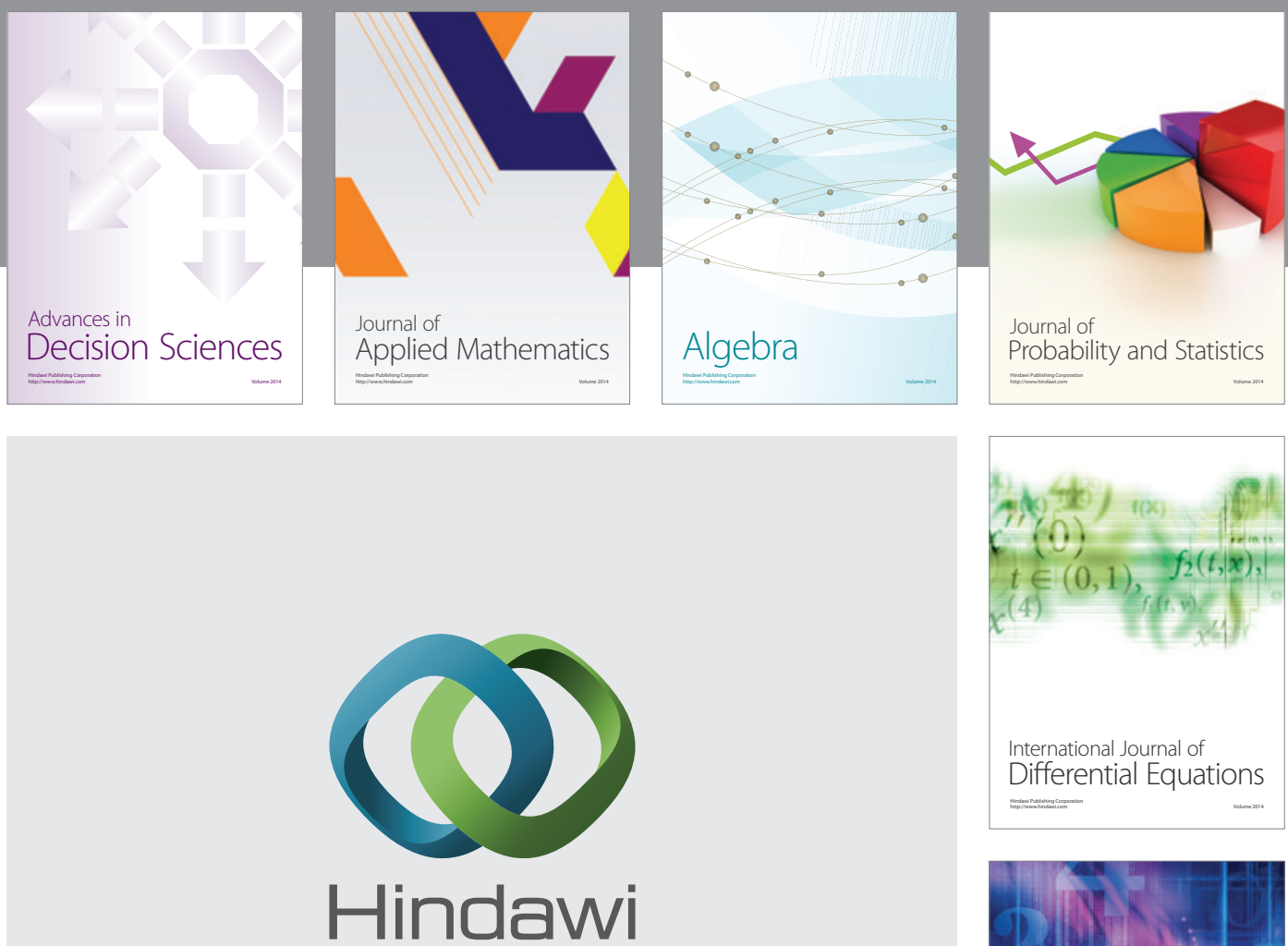

Submit your manuscripts at http://www.hindawi.com
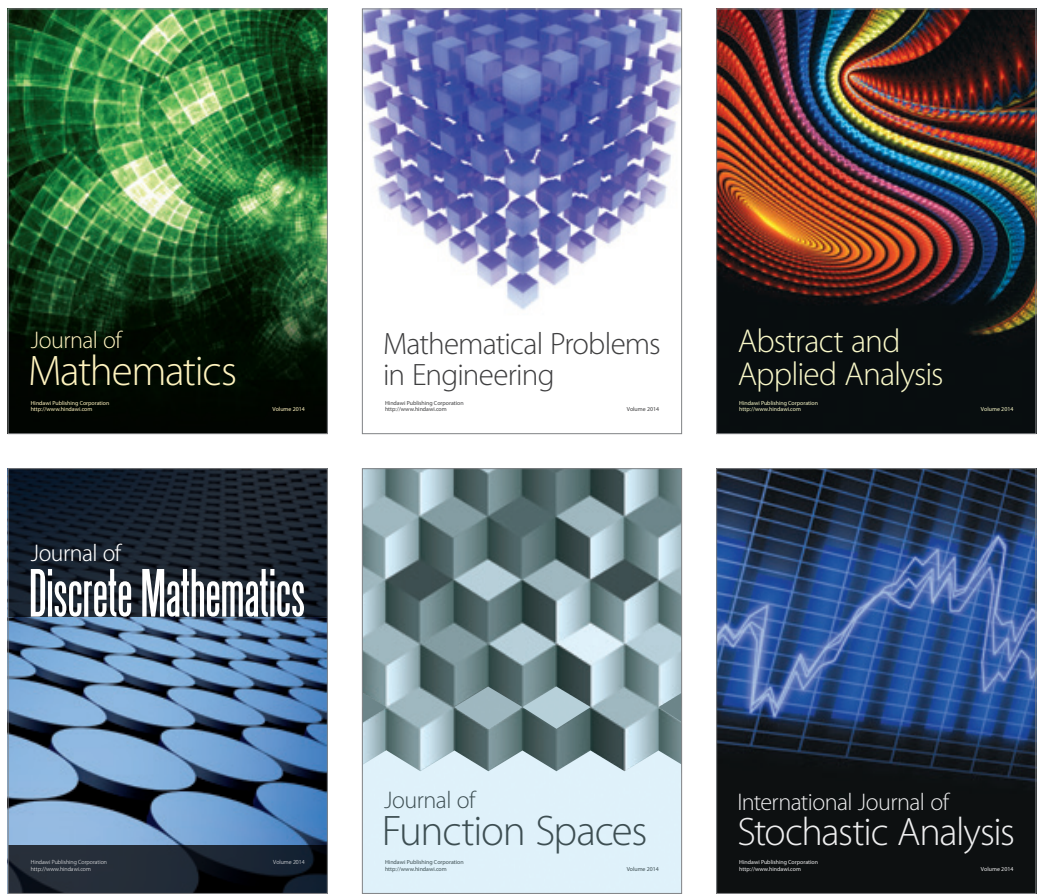

Journal of

Function Spaces

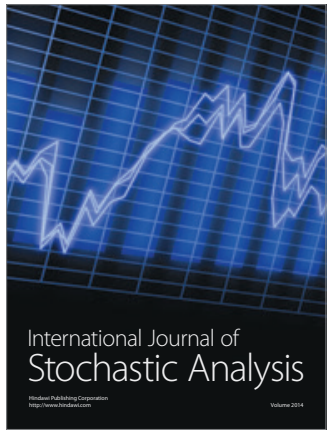

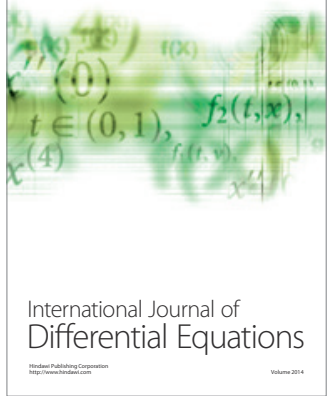
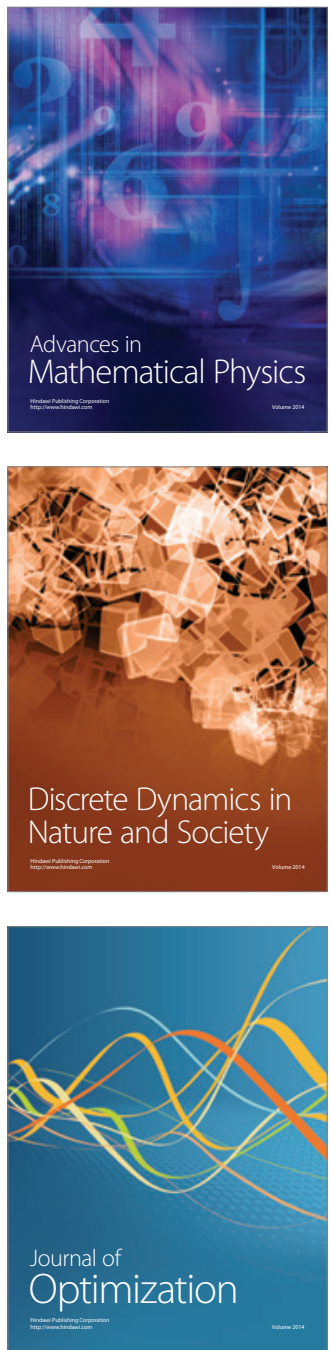